\title{
Las familias ante la encrucijada de la alfabetización mediática e informacional
}

\section{Families at the crossroads of media and information literacy}

\author{
Dercedes Romero Rodrigo \\ Departamento de Teoría de la Educación, Universidad de Valencia, España \\ m.mercedes.romero@uv.es \\ Cristina Gabarda Méndez \\ Área de Educación, Universidad Internacional de Valencia, España \\ cristina.gabarda@campusviu.es \\ Andrea Cívico Ariza \\ Área de Educación, Universidad Internacional de Valencia, España \\ andrea.civico@campusviu.es \\ (iD) Nuria Cuevas Monzonís \\ Área de Educación, Universidad Internacional de Valencia, España \\ nuria.cuevas@campusviu.es
}

\section{RESUMEN}

Ante una actualidad caracterizada por la hiperconexión, agentes como la escuela, la familia y los medios de comunicación continúan teniendo un papel muy relevante como responsables de la socialización de los menores. Las investigaciones hasta el momento han dado más cabida a las fricciones que ocurren entre estas dos realidades que al trabajo de cooperación que deben realizar para alcanzar un bien común. No obstante, tanto las familias como las escuelas deben ejercer una responsabilidad coordinada y coherente entre las mismas. Partiendo de esta realidad, este estudio analizará el papel de la familia como agente promotor para desarrollar el juicio crítico de los infantes en el contexto de la sociedad de la información. En este sentido, el objetivo de este estudio es ofrecer una serie de propuestas orientadas a las familias respecto a la educación activa de los menores en consonancia con los cinco ejes que propone la UNESCO para la educación mediática e informacional.

PALABRAS CLAVE alfabetización mediática, alfabetización informacional, educación en la vida familiar, tecnologías de la información.

\section{ABSTRACT}

Actualy, the hyper-connected reality in which we live, the main agents of socialization including the family, the school, and the media, continue to have an important role and responsible through socialization of minors. Research so far has focused more on the frictions that occur between these two realities than on the cooperative work they must do to achieve a com- 
mon good. Based on this reality, this study will analyse the role of the family as a promoter agent to develop children's critical judgement in the context of the information society. In this sense, the aim of this study is to offer a series of proposals aimed at families regarding the active education of children in line with the five axes proposed by UNESCO for media and information literacy.

KEYWORDS media literacy, information literacy, family life education, information technology.

\section{INTRODUCCIÓN}

La sociedad actual viene siendo definida por continuos e inmediatos cambios, los cuales transforman la realidad de las personas dirigiéndolas hacia una sociedad digital cada vez más hiperconectada (Blanco et al., 2020; Matesanz, \& Rios-Coello, 2021). En dicho contexto, juegan un papel crucial las Tecnologías de la Información y la Comunicación (TIC), favoreciendo múltiples canales informativos y comunicativos caracterizados por la interacción, la participación y la democracia que rige los mismos (Cabero, 2001; Cabero et al., 2019). Su impacto fue subrayado por la Unión Internacional de Telecomunicaciones (2010), enfatizando la necesidad de que la sociedad de la información avance y evolucione hasta convertirse también en la sociedad del conocimiento, la cual se basa en los principios de justicia política, social y económica para garantizar la dignidad de todas las personas. Sin ir más lejos, la tecnología juega un papel fundamental para el logro del cuarto Objetivo de Desarrollo Sostenible (ODS4) recogido en la Declaración de Qingdao (UNESCO, 2015). Tal es así, que este mismo organismo viene publicando directrices en la última década como lo fueron las Directrices para las políticas de aprendizaje móvil (West, \& Vosloo, 2013) o más recientemente las Directrices para la elaboración de políticas de recursos educativos abiertos (Miao et al., 2020). En este sentido, debemos entender las tecnologías no como un fin en sí mismo, sino como el medio más adecuado para desarrollar el conocimiento y promover la educación (Colomo et al., 2020). De este modo, constituyen un recurso poderoso a través del cual los seres humanos pueden adquirir nuevas competencias y destrezas para desenvolverse en la realidad descrita.

Las exigencias emergidas al albor de la era digital enfatizan la necesidad de la adquisición de habilidades y conocimientos propios, ampliando la tradicional concepción de persona analfabeta. Hoy por hoy lo digital forma parte de los alfabetismos básicos: desde saber usar la tecnología (Bortnik, 2020), navegar por Internet, crear y compartir hasta conocer las posibilidades que ofrecen las redes sociales (Busquet et al., 2020). Por tanto, el término de persona alfabetizada debe ser interpretado también desde la suma de todas aquellas alfabetizaciones envueltas por la tecnología y tan necesarias en la sociedad del siglo XXI (PérezEscoda et al., 2016). Desde esta premisa se explica la alfabetización en su sentido amplio como ya lo hicieron Gutiérrez-Martín y Tyner (2012), reconociendo la importancia que juegan diversos agentes educativos externos: desde los tradicionales medios de comunicación, Internet, redes sociales hasta los videojuegos, plataformas de contenido, aplicaciones u otros entornos virtuales con carácter más reciente.

A su vez, en un sistema educativo que no es ajeno a fenómenos como la apertura y exposición de las mismas propicia la convivencia de medios y escuela con anomalías de relevancia actual como lo son las noticias falsas (fake news) o la desinfodemia (UNESCO, 2020). Dichos retos subrayan la pertinencia de rescatar las propuestas en torno a la Alfabetización Mediática e Informacional (MIL) de los discentes. Entender los 
medios, comprender la información, analizar críticamente los contenidos y tomar decisiones conscientes y fundamentadas en torno a ellos "usándolos para satisfacer las necesidades de comunicación, expresión, formación o información que puedan generarse en diversas situaciones cotidianas" (García-Ruiz et al., 2014, p. 18) pero también contrarrestar los efectos ligados a la desigualdad de acceso y de creación, incluso de difusión de contenidos, resulta fundamental en la sociedad actual (UNESCO, 2015; 2020).

Y es en este punto donde convergen agentes educativos externos reseñados con la escuela y la familia, agentes de socialización primaria por excelencia. Tradicionalmente la investigación sobre esta cuestión se ha centrado más en indagar entorno a las fricciones con la institución escolar dejando en un segundo plano las referidas a la esfera privada o familiar. Como resultado existen diversidad de propuestas educativas encaminadas a contrarrestar los efectos negativos principalmente desde el contexto escolar, generalmente orientados al desarrollo de personas con juicio crítico. Es por esta razón por la que se ahondará acerca de dicho fenómeno en relación a las familias, a fin de ofrecer propuestas destinadas a los hogares, puesto que se trata de una responsabilidad compartida entre ésta y los centros, compromiso que exige colaboración y coherencia en la acción educativa de los menores (Egido, 2016).

\section{EQUIPAMIENTO TECNOLÓGICO Y USO DE LAS TECNOLOGÍAS: LA ANTESALA DE LA ALFABETIZACIÓN MEDIÁTICA E INFORMACIONAL DESDE LOS HOGARES}

Atendiendo a los datos acerca del equipamiento y uso de las TIC, según la encuesta del Instituto Nacional de Estadística acerca del equipamiento y uso de las TIC en el hogar (INE, 2020), el teléfono móvil está presente en prácticamente todos los hogares españoles. A su vez queda constatado que en los últimos años se ha consolidado un aumento considerable referido al uso de dispositivos tecnológicos por parte de los menores. La última estadística publicada indica que el ordenador es utilizado por el $91.5 \%$ de los menores, situando la utilización de Internet en el 94.5\%. Por otro lado, el $69.5 \%$ de la población de 10 a 15 años dispone de teléfono móvil.

Prestando atención a las diferencias por edad, el uso de TIC crece a medida que aumentan los años de los menores, sobre todo a partir de los 13. En cuanto a las diferencias por sexo, queda verificado que las menores realizan un uso mayor de las tecnologías, si bien es cierto que existen diferencias entre los tipos de actividades según los resultados del último informe publicado por el Instituto de la Juventud español. Todo parece indicar que el consumo en cuanto a series y películas online es similar entre los dos grupos, sin embargo, ellas hacen un uso mayor de Internet o redes sociales para la realización de tareas académicas mientras que ellos suelen recurrir a la red para jugar a videojuegos y chats (INJUVE, 2020). Hoy por hoy según esta misma fuente el $91.4 \%$ de los hogares españoles cuenta con acceso a Internet, siendo el porcentaje mayor si se contempla el uso que hacen los jóvenes (término que emplea también para referirse a los menores de edad en este caso) de Internet ampliando a otras conexiones más allá de las viviendas. Hoy en día todos los jóvenes utilizan Internet, el 99.8 \% según el INE (2020), destacando que la mitad de ellos hacen uso de las redes sociales para informase de política (INJUVE, 2020). En cuanto a la población adulta, el uso de Internet a partir de los 55 años se sitúa en el 89.5 \% y en el grupo de 65 a 74 años baja hasta el 69.7 \% (INE, 2020).

Teniendo en cuenta estos datos, se puede afirmar que la utilización de las TIC ha instaurado nuevos modos de comunicarse e interactuar socialmente, impactando en todos los ámbitos, desde la formulación 
de nuevas leyes que incluyan el uso adecuado de las TIC y la reglamentación sobre los delitos cibernéticos, como la organización de métodos para la enseñanza básica y superior en estudiantes, y como no, la configuración de estilos de crianza que abarquen el uso de dispositivos móviles (Perea et al., 2019). Sin embargo, y aunque la situación de confinamiento vivida por las familias el pasado año parece haber contribuido a la mejora de la valoración de lo digital en los hogares (Empantallados, 2020), las preocupaciones de los progenitores acerca de los hábitos, el tiempo de exposición a las pantallas, adicciones y el desarrollo de conductas inadecuadas en la red (entre otras) siguen estando presentes (Machuca-Rubio, \& Cabrera-Duffaut, 2020; Sánchez, 2021). Como puede observarse, el impacto de las tecnologías en la sociedad puede ser positivo y negativo sobre todo en función del uso que se haga de éstas, por ello es habitual que se establezcan medidas en ambas direcciones estudiadas.

Esta situación invita a reflexionar sobre si la población es lo suficientemente competente digitalmente como para hacer frente a este contexto. A tenor de los datos de la encuesta de equipamiento y uso de las TIC (INE, 2020), tan sólo el $44.1 \%$ de los usuarios de Internet posee habilidades avanzadas. En cuanto a la diferenciación por edad, el grupo con mayores habilidades es el de 16 a 24 años, lo que representa el $75.7 \%$ de la población. Por relación con la actividad, tienen mayor competencia digital el $83.3 \%$ de los estudiantes, el $50.5 \%$ de los ocupados y el $32.2 \%$ de los parados. Por consiguiente, la población joven posee una mayor competencia digital en comparación con la población adulta, lo cual invita a pensar que en numerosas ocasiones los progenitores desconocen aquello que los menores están realizando en las pantallas. Cada familia, desde su punto de vista, afronta esta situación de la mejor manera posible. Catalina y López de Ayala (2013) destacan que normalmente los padres suelen preguntar y observar cuando están conectados los hijos, cosa que hacen más del $67 \%$ de los padres. Tras esto, el siguiente paso sería la imposición de una temporalidad a la conexión y estar agregados al perfil de los hijos (58.8 \% y $41 \%$, respectivamente). En última instancia, puede aparecer la restricción de contenidos en Internet (13.6\%) o el seguimiento de las conversaciones, por menos del $7 \%$ de los padres. En línea con lo anterior, en una investigación reciente, las familias presentaron un interés moderado en la formación sobre gestión de los dispositivos móviles con menores (Ramírez-García et al., 2020), cuestión que no parece menor cuando este mismo colectivo reconoce que no está suficientemente preparado para hacer frente a la educación digital de sus hijos (Empantallados, 2018). De esta inquietud manifestada por las familias se constata la necesidad de trabajar por una alfabetización mediática con y para familias. Actuaciones que deben estar orientadas a proporcionar las destrezas necesarias para relacionarse crítica y eficazmente tanto con la tecnología como con los medios y sus mensajes. De esta manera, estarán en posición, por un lado, de poder supervisar y monitorizar aspectos que pudieran atañer riesgos y, por otro, de contribuir al desarrollo de la faceta mediática y de consumo responsable de los menores: especialmente en lo que respecta al acceso reflexivo y constructivo de la información y el conocimiento a través de los medios (González-Fernández et al., 2018).

\section{LA FAMILIA COMO AGENTE DE SOCIALIZACIÓN PRIMARIA}

Según Muñoz (2012), el fin de la familia como institución es lograr que las personas que la componen alcancen su plenitud humana. Por esta razón, en el contexto actual es necesario incidir en el uso crítico de la tecnología. Debido a ello, la familia debe actuar como mediadora en un contexto de sobreinformación digital, fomentando el uso crítico y reflexivo que los menores realizan de la tecnología (Torrecillas et al., 2017). 
El problema radica cuando la brecha digital existente entre los progenitores y los infantes por los diferentes niveles de competencias respecto al uso de las tecnologías es sustancial. Y es que son múltiples y diversos los servicios digitales y medios móviles a través de los cuales los menores interactúan con el contexto digital, exigiendo este hecho que las familias posean un conocimiento fundamentado de las TIC y de estrategias de mediación para conseguir una correcta alfabetización mediática e informacional de jóvenes e infantes.

El desarrollo personal de los seres humanos, siguiendo a Bronfenbrenner (2009), está influenciado por muchos aspectos y factores, destacando entre ellos los agentes de socialización como transmisores de los ideales y la cultura. Entre ellos se distingue por una parte los primarios: la familia ya señalada, pero también el grupo de pares y la escuela; $y$, por otra parte, los distales, haciendo referencia a los medios de comunicación. Estos, como agentes coeducadores, inciden en el desarrollo de la comprensión crítica de los menores, así como en la forma de interpretar y realizar juicios sobre lo que desean (Pallarès, \& Pañella, 2011) al generar situaciones y contextos en los que los individuos ponen en práctica la construcción de respuestas y acciones partiendo de su escala de valores. No obstante, cabe resaltar la importancia de que los mensajes que transmiten los diferentes agentes confluyan hacia la misma dirección, ya que los individuos constantemente reciben información sobre qué se espera de ellos en un futuro o quiénes son sin que exista una conciencia clara de quien genera el propio mensaje (Carrera et al., 2015). Por esta razón, son muchos los investigadores que abogan por la necesidad de que exista un trabajo colaborativo y cooperativo de todos los agentes socializadores (García, \& Martínez, 2006; Páez, 2015; Vázquez, \& López, 2014).

En este sentido, cabe destacar la importancia de la colaboración de la familia y la escuela como primer y segundo agente socializador para el correcto desarrollo identitario del infante (Cívico et al., 2019). La familia es la puerta de acceso del menor a la cultura que le rodea, aprendiendo de ella las normas, derechos y deberes para convivir en sociedad. Asimismo, siguiendo a Padilla et al., (2015), es "la responsable de diseñar el ambiente tecnológico en el que crecen sus hijos, junto con supervisar y mediar sus acciones” (p. 418). Esta función se complementa con el papel que juega la educación y el centro escolar. Y es que el sistema educativo es el primer ente a través del cual el individuo se sumerge en la sociedad, favoreciendo una socialización positiva al reproducirse la realidad social dentro del confort que el aula representa para el niño (Fernández, \& Rodríguez, 2002; Gracia, \& Musitu, 2000; Musitu, 2002; Ovejero, 2002; Palacios, 1999). Igualmente, el centro educativo es el responsable de crear un ambiente que estimule en su alumnado el empleo de las Tecnologías de la Información y la Comunicación (TIC), promoviendo una conciencia de uso responsable (Sasson, \& Mesch, 2017). De este modo, según los mismos autores, el contexto escolar presenta una doble finalidad: por un lado, satisfacer las necesidades individuales y sociales de los seres humanos; y por otro, afianzar los principales rasgos de la cultura, determinada actualmente por la revolución digital y tecnológica. Es en esta intersección en la que se originan buena parte de las desavenencias entre los subsistemas familiar/parental, educativo (representado por la escuela) y los jóvenes que, a pesar de pertenecer y estar integrados en cada uno de ellos, no supone impedimento alguno para revelarse. Los cambios originados por la tecnología son comprendidos y gestionados de manera diversa desde dichos grupos. Por ejemplo, en el caso de la escuela y la familia la preocupación respecto al uso inapropiado y dependiente de las TIC ha dado lugar a un continuo debate sobre las ventajas e inconvenientes sobre los diferentes dispositivos y aplicaciones. Entre los argumentos en contra, estos se presentan como elementos de distracción que pueden generar adicción respecto a su uso (Martínez, \& Capote, 2016), pudiendo producir consecuencias negativas en la construcción identitaria de los menores. En cambio, un uso adecuado y positivo los convierten en herramientas que fomentan nuevas formas de aprendizaje, además de incidir en el desarrollo social, cognitivo y afectivo de manera positiva (Romero, \& López, 2021). 


\section{DESAFÍOS, AMENAZAS Y OPORTUNIDADES DE LAS FAMILIAS ENTORNO A LA ALFABETIZACIÓN MEDIÁTICA E INFORMACIONAL DE LOS HIJOS}

Partiendo de la premisa de que la educación en los medios de comunicación es una necesidad imperante en la sociedad actual, se entiende que existe una gran responsabilidad dentro de las familias para poder adaptarse a estos nuevos desafíos en las sociedades digitales. Este objetivo no siempre es un hecho hito fácil de conseguir. Cabe no pasar por alto que la escuela y la familia son agentes determinantes en la adquisición de competencias mediáticas y en el propio proceso de alfabetización mediática, al ser considerados ambos los núcleos fundamentales de socialización tanto en los primeros años del individuo como durante su adolescencia y juventud. En esta sociedad, en la que las TIC y la conexión a Internet están totalmente integradas, se da la situación de familias que muestran inquietud por el panorama digital actual y la necesidad de, a través de formación comunicación y confianza, fortalecer las habilidades críticas de los menores y jóvenes en el uso de la red (Gamito et al., 2019).

Es una realidad que, en muchas ocasiones, la baja competencia parental por parte de las familias se convierte en un factor de riesgo ante conductas inapropiadas en el uso de Internet como las ciber agresiones por parte de los jóvenes, tanto a sus iguales, como a sus parejas (Cortés et al., 2019; Gabarda, 2020; Garaigordobil, \& Machimbarrena, 2017). Otro factor a tener en cuenta dentro de las familias, vinculado al tema de alfabetización digital y mediática es que, puede darse una situación de brecha digital entre los diferentes perfiles intergeneracionales que componen las familias. El alumnado actual en las sociedades de pantallas son nativos digitales y viven la tecnología como parte de su entorno natural (Prensky, 2009). Es por esta razón por la que supone un reto para las familias el llevar a cabo una adecuada alfabetización mediática de los hijos en esta era digital presente.

Atendiendo a uno de los últimos estudios sobre alfabetización mediática en escenarios familiares, concretamente al estudio realizado por González-Fernández et al. (2019), es reseñable los resultados que aportan en este sentido, como muestra el amplio conocimiento que tienen las familias sobre los medios de comunicación, así como el temor hacia el uso y la construcción de mensajes en las redes sociales y la necesidad de una mayor formación en torno a los mismos. Dichos autores consideran necesario el avance urgente en el diseño de planes formativos de alfabetización mediática potenciadores de habilidades críticas en el entorno familiar que fomenten la participación de sus miembros como prosumidores responsables, dotados de conocimientos sobre lenguaje técnico y audiovisual de los medios, manejo práctico y responsable, influencia de valores y tendencias estéticas de los medios.

Es bien sabido, ya en plena era tecnológica, que la tecnología de la información plantea dos claras opciones de uso. Por un lado, entre sus múltiples beneficios y bondades, destaca el amplio acceso a la información, las variadas opciones de ocio y recreativas y, por otro, las innumerables posibilidades comunicativas y el acceso a distintos modos de aprendizaje y enseñanza. Siendo esto así cabe no ignorar la cara menos amable, oscura y peligrosa de las TIC, destacando el alto número de jóvenes expuestos por ejemplo a material sexual de manera involuntaria (Gil-Juliá et al., 2018) e, incluso, está muy presente el riesgo de adicción que puede generar el uso excesivo y no controlado de las mismas (Muñoz-Miralles et al., 2014; Ruiz-Palmero et al., 2016). Relevante es tener en cuenta, que las tecnologías presentan también usos como el maltrato, amenazas y agresiones (Castro, 2017). La literatura científica al respecto aporta datos sobre el conocimiento de los riesgos percibidos por 
los jóvenes respecto a Internet, estos mismos manifiestan ser conocedores de dichos peligros y valoran sus capacidades en el ámbito digital como buena, confiando es sus acciones a través del ámbito digital (GarcíaValcárcel et al., 2018) pero, a su vez, los mismos estudiantes, una vez evaluados, presentan bajos resultados en cuanto a las habilidades relacionadas con la seguridad en Internet (Garmendia et al., 2017). Otro factor que debe tenerse en cuenta es que el acceso de estos menores a las nuevas tecnologías se realiza habitualmente de manera espontánea e informal (Scroeder, 2018) antes de que hayan tenido la oportunidad de desarrollar habilidades que les permitan manejar los riesgos vinculados a las mismas (Livingstone, 2018). Téngase en cuenta que la época adolescente se caracteriza, entre otros, por un bajo control de impulsos, falta de madurez y, una posible inclinación a comportamientos inadecuados. Estos factores pueden contribuir a aumentar su vulnerabilidad ante los peligros de las nuevas tecnologías convirtiéndolos en el colectivo más vulnerable en el contexto digital y mucho más cuando se expone sin ningún tipo control o supervisión parental, escolar o social (Argente et al., 2017; Besoli et al., 2018; Donoso et al., 2018; Gamito et al., 2019).

Diversos estudios coinciden en que el núcleo familiar es esencial en la educación de valores y competencias de cara a una alfabetización mediática eficaz. Así pues, se hace necesario seguir realizando tareas que fomenten el desarrollo de la mediación parental proactiva, tal y se abordará más adelante. Por lo que, las familias, deben ejercer una labor de cuidado y mediación parental en el uso de las TIC para prevenir, entre otros, problemas de acoso a través de las redes sociales (Gabarda, 2020). De hecho, se ha comprobado en diversos estudios que, la supervisión y mediación digital parental/familiar, es un factor de protección frente a la aparición de conductas inapropiadas en Internet (Gabarda, 2020; Rial, \& Gómez, 2018). Esta mediación parental, para que sea eficaz, debe ser proactiva, es decir, que los padres o tutores sean capaces de promover las conductas seguras y responsables a través de una comunicación eficaz con los menores, posibilitando, de este modo la que se promueva la alfabetización digital y la adquisición de habilidades críticas para la evitación de riesgos en Internet. Esta mediación proactiva debe ser entendida como una serie de acciones o comportamientos dirigidos a la selección, uso y consumo de los medios, que no se limite únicamente a al establecimiento de control parental, sino que sea necesaria una adecuada formación padres y tutores que permita una mayor fluidez en la comunicación familiar y formule retos en el desarrollo de capacidades y valores individuales (González-Fernández et al., 2019). En esta línea, el estudio realizado por Gamito et al. (2019), señala la alfabetización y educación digital como un buen instrumento de empoderamiento y la opción más coherente a largo plazo, a pesar de que a veces el ritmo tecnológico, la desactualización digital de las familias y la falta de formación docente dificulta la mediación adulta en la realidad digital de la población menor de edad, por lo que debe fortalecerse la formación tanto de carácter técnico como crítico a las familias.

\section{PROPUESTAS DESTINADAS A LAS FAMILIAS DESDE UNA PERSPECTIVA EDUCATIVA}

Las propuestas educativas que son presentadas a continuación pretenden ser un punto de partida para implementar y desarrollar competencias mediáticas e informacionales desde el ámbito familiar. Son el resultado del análisis del contexto tecnológico descrito en el marco del hogar, así como de los desafíos, amenazas y oportunidades de las familias identificadas en los apartados anteriores. Con el propósito de abordar su exposición de manera integral, se parte de la propuesta de la UNESCO en torno a los cinco ejes más relevantes de la educación mediática (UNESCO, 2015; 2020) con objetivo de responder a cada uno de los retos que plantean. 
El primero de los ejes señala que los medios, la información y la comunicación (independientemente del formato, soporte o canal) están al servicio de la sociedad y del desarrollo sostenible. Por tanto, en la sociedad actual reconocer y aceptar la importancia que juega la información y los medios que la hacen accesible invita a estar vigilantes, puesto que como se ha podido constatar en multitud de ocasiones, no siempre ha contribuido al desarrollo sostenible. Comprender las funciones e intereses de los medios en la alfabetización mediática de la ciudadanía, más cuando la difusión hoy por hoy es instantánea, no está exenta de riesgos y efectos adversos ligados a la desinformación, es una cuestión que igualmente debe ser abordada en el ámbito familiar (Gabarda, 2020, Gamito et al., 2019). Desde este espacio es preciso conocer y enseñar a utilizar fuentes de información diversa, incluyendo tradicionales (prensa, televisión y bibliotecas) y digitales (más allá de las redes sociales, como pueden ser las alternativas digitales de los referidos medios tradicionales). Familiarizarse en su uso es un paso necesario para reducir la brecha digital con respecto a los menores: conocer para saber, saber para tutelar. A su vez, participar del debate público surgido en la red y hacerlo con los hijos: compartir espacios para el conocimiento, contraste de información, apertura a opiniones y posicionamientos diversos son algunos ejemplos para poder empezar a trabajar en esta dirección.

En segundo orden y teniendo en cuenta que cualquier ciudadano es transmisor y creador de mensajes y contenido, una persona alfabetizada no se circunscribe a saber localizar ciertos contenidos, acceder a medios o navegar en plataformas diversas. Además de vislumbrar las funciones e intereses de los medios y su contexto, es preciso desarrollar la capacidad de evaluar los mensajes, interpretar y hacer propia la información antes de poder generar contenido propio adecuadamente. Téngase en cuenta que, tras la evolución de la Web 1.0 a la 2.0, la posibilidad de ser usuarios activos está en la mano de cada uno de los usuarios. Además, en la actualidad, el fenómeno comunicativo está evolucionando ofreciendo nuevas posibilidades ligadas al mundo de los sentidos: usuarios y creadores se mueven cada vez más en un plano de identificación emocional. Dichas sensaciones, emociones y por qué no, sentimientos, se convierten en un preciado bien sobre el que conectar relaciones y experiencias con finalidades diversas, siendo clave tomar conciencia acerca de la libertad que se cree poseer y que se posee en realidad a la hora de tomar decisiones. Cabe no pasar por alto que la comunicación tiene un componente emocional y que, en muchas ocasiones, los medios pretenden provocar una respuesta en este plano que afiance determinadas acciones o conductas. Por esta razón, desde los hogares, compartir y debatir en cuanto a las percepciones y evocaciones de mensajes naturalizados, reflexionar entre el deseo ligado a una necesidad real o sembrada bajo las tendencias de mercado contribuirá a educar hijos e hijas conscientes de sus patrones de consumo y relación con los medios. Educar para cultivar una actitud abierta con respecto a los medios e intereses manifiestos y ocultos, no solamente contribuirán al desarrollo de ciudadanos activos y críticos, sino también creativos a la hora de dar respuesta a situaciones de injusticia o vacíos en los que la sociedad civil se organiza y da respuesta justamente apoyándose en estos medios. Siendo esto así, crear y publicar contenido en colaboración con los adultos o bajo su tutela es el principio para enseñar a hacer un uso responsable de la red minimizando los riesgos que ésta presenta (Cívico et al., 2019). En una sociedad saturada de mensajes, donde la comunicación y sobre abundancia de mensajes invade tanto la esfera pública como la privada, participar creando, compartiendo y debatiendo es capital para avanzar en una ciudadanía libre, plena ¿por qué no empezar a hacerlo amparados por la seguridad y confianza de la familia? 
En cuanto al tercer eje, ligado a la no neutralidad de informaciones, mensajes y conocimientos, cabe adquirir consciencia sobre dicho fenómeno, así como herramientas para hacerle frente (Sasson, \& Mesch, 2017). En ocasiones, descifrar la falsa neutralidad de ciertos mensajes puede resultar complejo. Por esta razón desde la cotidianidad del hogar, acceder a fuentes diversas en cuanto a posicionamientos ideológicos invita a conocer posturas, pero también a encontrarse en el otro. En un contexto polarizado, donde el sesgo de confirmación está a la orden del día, cuestionar en el hogar las razones tras las que se viralizan ciertas noticias y no otras, el papel que juega cada uno para que sea así y enseñar a acudir a canales oficiales para contrastar determinada información son algunos ejemplos de los deberes de la familia como agente de socialización primaria en el tema que nos ocupa. No hay mejor herramienta que educar desde el ejemplo, favoreciendo los espacios de comunicación y debate con los menores, invitándoles a expresarse libremente sin olvidar que para hacerlo correctamente el saber, es decir, el conocimiento más allá de la información que comparten los medios, es esencial para tener un verdadero juicio crítico.

En cuarto lugar, los ciudadanos tienen derecho a no verse comprometidos por la espiral mediática e informacional. En este entramado de acciones para el desarrollo de una alfabetización mediática e informacional en el ámbito familiar, la ética y la cautela también son pilares sobre los que conviene incidir. Por un lado, porque resulta primordial desarrollar actitudes cívicas propias con respecto al uso de medios, creación y difusión de contenidos incluyendo cómo relacionarse con los demás (Torrecillas et al., 2017); el respeto y la tolerancia en relación al que piensa diferente no pueden pasar a un segundo plano por la posibilidad de anonimato en la red. Por otro, y siendo sujetos activos, cuidar la propia imagen, enseñar a tomar conciencia de lo que hacen respecto a la actividad con los medios y la red. Prácticamente cualquier acción deja una huella por lo que, tanto tomar conciencia como hacerles partícipes de sus derechos y obligaciones, enseñándoles a protegerse de los riesgos que entraña el mundo virtual, es importante. La falsa sensación de seguridad, sumado al rechazo a los padres como figuras de autoridad por parte de algunos menores durante de la adolescencia, la búsqueda de referentes de éxito ligados a la inmediatez y que muestran la cara amable de los medios pueden llevar a los jóvenes a desarrollar actitudes poco sanas en relación al consumo de medios. Esta es una de las razones por las que trabajar desde el hogar la necesidad de desconexión digital en todos los sentidos, progenitores incluidos, para educar con el ejemplo sin relaciones de dependencia marcadas por el abuso de consumo de medios y tecnología que los soporta.

Para finalizar, tal y como viene reflejado en la quinta ley de alfabetización mediática e informacional, se trata de un proceso que precisa de tiempo y constancia por parte de los progenitores (Gamito et al., 2019; González-Fernández et al., 2019). En un entorno altamente volátil, los conocimientos y habilidades adquiridas quedan desfasados en un margen de tiempo nada desdeñable, si bien es cierto que las jóvenes generaciones parecen adaptarse a ellos con mayor flexibilidad y rapidez que sus mayores. Probablemente, en el campo de las nuevas habilidades o competencias digitales que surjan del resultado de la evolución constante de los medios, los jóvenes se sitúen en mejor posición. No obstante, los adultos cuentan con el saber y saber ser que les permitirá desarrollarse de manera completa, cultivando la autonomía moral con respecto al uso, consumo y disfrute de los medios e información, velando por un desarrollo consciente y responsable tanto para sí mismos como en su relación con los demás. 


\section{REFERENCIAS}

Argente, E., Vivancos, E., Alemany, J., \& García-Fornes, A. (2017) Educando en privacidad en el uso de redes sociales. Educa tion in the Knowledge Society (EKS), 18(2), 107-126. https:// doi.org/10.14201/eks2017182107126

Besoli, G., Palomas, N., \& Chamarro, A. (2018). Uso del móvil en padres, niños y adolescentes: Creencias acerca de sus riesgos y beneficios. Aloma Revista de Psicología, Ciènces de l'Educació i de l'Esport, 36(1), 29-39. https://doi. org/10.51698/aloma.2018.36.1.29-39

Blanco, I., Fernández, L. M., \& Suárez, R. (2020). Vulnerabilidad y cultura digital. Riesgos y oportunidades de la sociedad hiperconectada. Dykinson.

Bortnik, S. (2020). Guía para la crianza en un mundo digital: Cómo educar para un uso sano y seguro de la tecnología. Siglo XXI Editores.

Bronfenbrenner, U. (2009). The ecology of human development: Experiments by nature and design. Harvard University Press.

Busquet, J., Calsina, M., Medina, A., \& Flaquer, L. (2020). 262 conceptos clave de sociología: Nueva edición revisada y ampliada. Editorial UOC.

Cabero (2001). Nuevas tecnologías aplicadas a la educación. Síntesis.

Cabero, J., Torres, L., \& Hermosilla, J. M. (2019). Las TIC y la creación de una ciudadanía crítica-digital. Education in the Knowledge Society (EKS), 20, 22-10. https://doi.org/10.14201/ eks2019 20 a22

Carrera, M.V., Lameiras, M., Cid, X.M., Rodríguez, Y., \& Alonso, P. (2015). De las tecnologías del género a las tecnologías de la subversión, un desafío pedagógico para la educación social. Revista de Educación Social, 21, 8-27.

Castro, C. (2017). Violencia a través de las TIC: el ciberacoso en escolares de la Comunidad Autónoma de Madrid [Tesis Doctoral]. Universidad Pontificia Comillas de Madrid, Madrid, España. https://repositorio.comillas.edu/xmlui/bitstream/ handle/11531/20850/TD00276.pdf?sequence $=1$ \&isAllowed $=y$
Catalina, B., \& López de Ayala, M. C. (2013). Los padres ante el uso de Internet y redes sociales por menores. Control y protección. Actas del V Congreso Internacional de Comunicación Social. Universidad de La Laguna, La Laguna, España. http://www.revistalatinacs.org/13SLCS/2013 actas/077 Catalina.pdf

Cívico, A., González, E., \& Colomo, E. (2019). Análisis de la percepción de valores relacionados con las TIC en adolescentes. Revista Espacios, 40(32), 18

Colomo, E., Sánchez, E., Ruíz, J., \& Sánchez, J. (2020). Percepción docente sobre la gamificación de la evaluación en la asignatura de Historia en educación secundaria. Información tecnológica, 31(4), 233-242. http://dx.doi.org/10.4067/S0718$\underline{07642020000400233}$

Cortés, A. F. M., De los Ríos, O. L. H., \& Pérez, A. S. (2019). Factores de riesgo y factores protectores relacionados con el ciberbullying entre adolescentes: Una revisión sistemática. Papeles del psicólogo, 40(2), 109-124. https://doi.org/10.23923/pap. psicol2019.2899

Donoso, T., Rubio, J., \& Vilà, R. (2018). La adolescencia ante la violencia de género 2.0: concepciones, conductas y experiencias. Educación XX1, 21(1), 109-134. https://doi.org/10.5944/ educxx1.20180

Egido, I. (2016). Las relaciones entre familia y escuela. Una visión general. Participación Educativa. Revista del Consejo Escolar del Estado, 4(7), 11-18.

Empantallados. (2018). El impacto de las pantallas en la vida familiar. Hogares hiperconectados: el comportamiento de padres e hijos en un entorno multipantalla. https://empantallados. com/especiales/estudio2018/

Empantallados. (2020). El impacto de las pantallas en la vida familiar durante el confinamiento. https://empantallados.com/ covid19/

Fernández, L., \& Rodríguez, F. J. (2002). La prevención de la violencia: Hechos y mitos. Psicothema, 14(1), 147-154. 
Gabarda, C. (2020). Violencia en la red en población adolescente: Cyberbullying y Ciberviolencia de Pareja desde el perfil del agresor [Tesis Doctoral]. Universidad de Valencia, Valencia, España. https://mobiroderic.uv.es/handle/10550/75201

Gamito, R., Aristizabal, P., \& Vizcarra, M. T. (2019). Sociedad multipantalla: un reto educativo para familia y escuela. Revista Prisma Social, (25), 398-423.

Garaigordobil, M., \& Machimbarrena, J.M. (2017). Stress, competence, and parental educational styles in victims and aggressors of bullying and cyberbullying. Psicothema, 29(3), 335340. https://doi.org/10.7334/psicothema2016.258

García, F.J., \& Martínez, R.A. (2006). La relación entre los centros escolares, las familias y los entornos comunitarios como factor de calidad de la educación de menores y adultos. Cultura y Educación, 18(3-4), 213-218. https://doi. org/10.1174/113564006779173019

García-Ruiz, R., Gozálvez, V., \& Aguaded, I. (2014). La competencia mediática como reto para la educomunicación: instrumentos de evaluación. Cuadernos.info, (35), 15-27. http://dx.doi. $\operatorname{org} / 10.7764 /$ cdi.35.623

García-Valcárcel, A., Olmos, S., Sánchez, J. C., \& Cabezas, M. (2018). ¿Hacen los adolescentes un uso seguro de Internet? [Comunicación] XXVI Jornadas Universitarias de Tecnología Educativa (JUTE), San Sebastián, España. https://www.dropbox.com/s/j9vqqft5blupkrw/JUTE18 25.pdf?dl=0

Garmendia, M., Jimenez, E., Casado, M.A., Garitaonandia, C., \& Mascheroni, G. (2017). Net Children Go Mobile. Riesgos y Oportunidades en internet y uso de dispositivos móviles entre menores españoles (2010-2015). Red.es, Universidad del País Vasco/Euskal Herriko Unibertsitatea.

Gil-Juliá, B., Castro-Calvo, J., Ruiz-Palomino, E., García-Barba, M., \& Ballester-Arnal, R. (2018). Consecuencias de la exposición involuntaria a material sexual en adolescentes. Revista INFAD de Psicología. International Journal of Developmental and Educational Psychology, 1(1), 33-44. https://doi. org/10.17060/ijodaep.2018.n1.v2.1159

González-Fernández, N., Ramírez, A., \& Aguaded, I. (2019). Alfabetización mediática en escenarios familiares. Diagnóstico, necesidades y propuesta formativa. Education in the Knowled- ge Society (EKS), 20(1), 185-197. https://doi.org/10.14201/ eks2019 20 a11

González-Fernández, N., Ramírez-García, A., \& Salcines-Talledo, I. (2018). Competencia mediática y necesidades de alfabetización audiovisual de docentes y familias españolas. Educación XXI, 21(2), 301-321. https://doi.org/10.5944/educxx1.16384

Gracia, E., \& Musitu, G. (2000). Psicología Social de la familia. Paidós.

Guitiérrez-Martín, A., \& Tyner, K. (2012). Educación para los medios, alfabetización mediática y competencia digital. Revista Comunicar, 19(38), 31-39. https://doi.org/10.3916/C38-2012-02-03

INJUVE (2020). Informe Juventud en España 2020. Instituto de la Juventud. http://www.injuve.es/sites/default/files/adjuntos/2021/03/informe juventud espana 2020.pdf

Instituto Nacional de Estadística (2020). Encuesta sobre Equipamiento y Uso de Tecnologías de Información y Comunicación en los Hogares. https://www.ine.es/prensa/tich 2020.pdf

Livingstone, S. (2018). La vida online de la infancia. En E. Jiménez, M. Garmendia, y M. A. Casado (Coords.), Entre selfies y WhatsApps. Oportunidades y riesgos para la infancia y la adolescencia conectada (pp. 13-29). Gedisa.

Machuca-Rubio, J. B., \& Cabrera-Duffaut, A. (2020). Percepción de la exposición en seguridad informática de los niños y adolescentes durante la pandemia COVID-19. Polo del Conocimiento: Revista científico - profesional, 5(1), 37-51. http://dx.doi. org/10.23857/pc.v5i1.1874

Martínez, A., \& Capote, C. (2016). Las tecnologías de la información y la comunicación (TIC) en la educación: análisis descriptivo y su relación con personalidad e inteligencia emocional. En J.L Castejón (Coord.), Psicología y Educación: presente y futuro (pp. 1338-1339). ACIPE.

Matesanz, C., \& Rios-Coello, Y. (2021). La construcción del espacio privado en la modernidad líquida: el cuarto propio conectado. Arte, individuo y sociedad, 33(1), 217-235. https://dx.doi. org/10.5209/aris.67954

Miao, F., Mishra, S., Orr, D., \& Janssen, B. (2020). Directrices para la elaboración de políticas de recursos educativos abiertos. UNESCO. https://unesdoc.unesco.org/ark:/48223/pf0000373558 
Muñoz, A. (2012). Puntos de inflexión y recuperación del desarrollo infantil tras experiencias iniciales de adversidad. Escritos de psicología, 5(1), 1-8.

Muñoz-Miralles, R., Ortega-González, R., Batalla-Martínez, C., López-Morón, M. R., Manresa, J. M., \& Torán-Monserrat, P. (2014). Acceso y uso de nuevas tecnologías entre los jóvenes de educación secundaria, implicaciones en salud. Estudio JOITIC. Atención Primaria, 46(2), 77-88. https://doi. org/10.1016/j.aprim.2013.06.001

Musitu, G. (2002). Las conductas violentas de los adolescentes en la escuela. El rol de la familia. Aula Abierta, (79), 109-138.

Ovejero, A. (2002). Cultura de la pobreza: violencia, inmigración y fracaso escolar en la actual sociedad global. Aula Abierta, (79), $71-84$

Padilla, S., Rodríguez, E., Álvarez, M., Torres, A., Suárez, A., \& Rodrigo, M. J. (2015). La influencia del escenario educativo familiar en el uso de Internet en los niños de primaria y secundaria. Infancia y Aprendizaje, 38(2) 402-434. https://doi.org/1 $\underline{0.1080 / 02103702.2015 .1016749}$

Páez, R.M. (2015). Familia y escuela: una alianza necesaria en la gestión escolar. Revista iberoamericana de educación, 67, 159-180. https://doi.org/10.35362/rie670228

Palacios, J. (1999). La familia y su papel en el desarrollo afectivo y social. En F. López, I. Etxebarría, M.J. Fuentes, M.J. Ortíz (Comps), Desarrollo afectivo y social (pp. 267-285). Pirámide.

Pallarès, A.A., \& Pañella, R.B. (2011). El cine en la escuela: Propuestas didácticas de películas para primaria y secundaria. Graó.

Perea, E. P., Torres, B. D., \& Zuluaga, L. M. (2019). De TIC a TAC una propuesta de educación para padres y adolescentes sobre el uso de las tecnologías de la información y la comunicación. [Tesis de Grado]. Universidad CES de Medellín, Medellín, Colombia. https://repository.ces.edu.co/bitstre am/10946/4776/3/1152466072 2019.pdf

Pérez-Ecoda, A., Aguaded, I., \& Rodríguez-Conde, Mª J. (2016). Generación digital v.s. escuela analógica. Competencias digitales en el currículum de la educación obligatoria. Digital Education Review, (30), 165-183.
Prensky, M. (2009). H. sapiens digital: From digital immigrants and digital natives to digital wisdom. Innovate: journal of online education, 5(3), 1-9.

Ramírez-García, A., Salcines-Talledo, I., \& Fernández-González, N. (2020). Los dispositivos móviles en el hogar. Interés formativo de las familias españolas. Revista Española de Orientación y Psicopedagogía, 31(1), 43-61. https://doi.org/10.5944/reop. vol.31.num.1.2020.27286

Rial, A., \& Gómez, P. (2018). Adolescentes y uso problemático de Internet. Claves para entender y prevenir. En E. Jiménez, M. Garmendia, y M. A. Casado (Coords.), Entre selfies y WhatsApps. Oportunidades y riesgos para la infancia y la adolescencia conectada (pp. 157-172). Gedisa.

Romero, M., \& López, M. (2021). Luces, sombras y retos del profesorado entorno a la gamificación apoyada en TIC: un estudio con maestros en formación. Revista Electrónica Interuniversitaria de Formación del Profesorado, 24(2), 167-179. https:// doi.org/10.6018/reifop.470991

Ruiz-Palmero, J., Sánchez-Rodríguez, J., \& Trujillo-Torres, J. M. (2016). Utilización de Internet y dependencia a teléfonos móviles en adolescentes. Revista Latinoamericana de Ciencias Sociales, Niñez y Juventud, 14(2), 1357-1369.

Sánchez, I. M. (2021). Impacto psicológico de la COVID-19 en niños y adolescentes. MEDISAN, 25(1), 123-140.

Sasson, H., \& Mesch, G. (2017). The Role of Parental Mediation and Peer Norms on the Likelihood of Cyberbullying. Journal of genetic psychology, 178(1), 15-27. https://doi.org/10.1080/0022 1325.2016.1195330

Scroeder, R. (2018). Towards a theory of digital media. Information Communication and Society, 21(3), 323-339. https://doi. org/10.4135/9781473910805.n8

Torrecillas, T., Vázquez, T., \& Monteagudo, L. (2017). Percepción de los padres sobre el empoderamiento digital de las familias en hogares hiperconectados. El profesional de la información, 26(1), 97-104. https://doi.org/10.3145/epi.2017.ene.10

UNESCO (2015). Declaración de Qingdao. Aprovechar las oportunidades digitales. Liderar la transformación de la educación. https://unesdoc.unesco.org/ark:/48223/pf0000233352 
Innoeduca. International Journal of Technology and Educational Innovation

Romero Rodrigo, M., Gabarda Méndez, C., Cívico Ariza, A., Cuevas Monzonís, N.

UNESCO (2020). Declaración de Seúl sobre la Alfabetización mediática e informacional para todos y por todos: Una defensa contra las desinfodemias. https://en.unesco.org/sites/default/files/seoul declaration mil disinfodemic es.pdf

Unión Internacional de Telecomunicaciones (2010). Informe sobre el Desarrollo Mundial de las Telecomunicaciones/TIC de 2010. Verificación de los objetivos de la CMSI. Place des Nations.

Vázquez, C., \& López, S. (2014). Creencias sobre la relación familia-escuela. Cambios en el futuro profesorado tras recibir formación específica. Revista de Estudios e Investigación en Psicología y Educación, 1(2), 111-121. https://doi.org/10.17979/ reipe.2014.1.1.35

West, M., \& Vosloo, S. (2013). Directrices para las políticas de aprendizaje móvil. UNESCO. https://unesdoc.unesco.org/ ark:/48223/pf0000219662 